\title{
Pluractionality and the unity of the event
}

\author{
Lucia M. Tovena \\ Université Paris VII
}

\begin{abstract}
This paper exposes shortcomings of an analysis to singleevent plural verbs (Cusic's event-internal pluractionals) based on temporal discontinuity. It shows how to ground discontinuity on the participant used to measure out the event, by forcing breaches in the property of Mapping-to-SubObject that its theta role should have. This provides an explanation for why phases cannot be described by the same predicate applying to the whole event, which in turn exposes differences with respect to semelfactive verbs and the minimal units they are made of.
\end{abstract}

\section{Introduction}

The Italian sentence in (1a) describes the eating of one apple by Luisa that differs from the event described by (1b) because it takes place on and off. Culmination is an implicature in both sentences.
a. Luisa ha mangiucchiato la mela
'L. ate the apple'
b. Luisa ha mangiato la mela
'L. ate the apple'

Mangiucchiare is an event-internal pluractional verb [8][1][14], and the multiplicity of subevents it describes, aka 'phases' [1], does not impact on the singularity of the event or of its participants. On the contrary, event-external pluractional verbs denote pluralities of units higher up in Cusic's hierarchy, namely 'events' or 'occasions'. Lasersohn's [7] definition in (2) concerns the latter. Sources of multiplicity are subsequent times, distinct places or participants, which can be seen as the key in a form of distribution where the event predicate would be the share. Such a key is provided in (2) by a non-overlap condition and a function $f$ that is a temporal or spatio-temporal trace, or a thematic role. Lasersohn adds the constraints $\mathrm{P}=\mathrm{V}$ unless $\mathrm{X}$ is a plurality of phases, and $n \geq 2$ on cardinality, that relies on pragmatic contextual information for fixing the value of $n$.

(2) $\quad \mathrm{V}-\mathrm{PA}(\mathrm{X}) \Leftrightarrow \forall$ e e' $\in \mathrm{X}\left[\mathrm{P}(\mathrm{e}) \& \neg \mathrm{f}(\mathrm{e}) \circ \mathrm{f}\left(\mathrm{e}^{\prime}\right)\right] \& \operatorname{card}(\mathrm{X}) \geq \mathrm{n}$

$\mathrm{PA}=$ pluractional affix, $f$ is a temporal/spatio-temporal trace function or a thematic role assigned by $\mathrm{V}$

Cases like (1a) are not covered by (2) precisely because multiplicity has an event-internal source, cf. [15] for criticisms. Recently, two proposals have 
been put forth for this and similar cases. One seeks to identify phases via their temporal trace [11], the other via their local participants [12;13; 15]. This paper discusses shortcomings in grounding phases on time and shows how to ground them on the participant that is used to measure out the event. This provides an explanation for why phases cannot be described by the same predicate applying to the event and how they differ from minimal units of an event.

\section{Event-internal plurality}

Tatevosov has argued that the morpheme -kala- in Chuvash (Altaic, Turkic) introduces a form of verb plurality arising from discontinuity of subevents in a single event. ${ }^{1}$ This morpheme is analysed as a degree modifier that lowers below standard a contextually determined gradable property of an event predicate.

$$
\begin{aligned}
& \|- \text { kala }-\|=\lambda \mathrm{P} \lambda \mathrm{e} \exists \mathrm{d}\left[\mathrm{F}_{c}(\mathrm{P})(\mathrm{e})=\mathrm{d} \wedge \mathrm{d}<\operatorname{STANDARD}\left(\mathrm{F}_{c}\right)(C)\right] \\
& \mathrm{F}_{c}=\text { variable over degree functions specifying the degree of a grad- } \\
& \text { able property of an event, } \mathrm{P}=\text { type of event, } C=\text { comparison class }
\end{aligned}
$$

Continuity is the property discussed, and $\mathrm{F}_{c}$ in (3) is assigned the function $\mathrm{F}_{\text {Continuity }}$ as a value. The standard of comparison for the scale of continuity is the maximal degree, because this is an upper closed scale. A function $\tau^{C}$, called COVERING TIME, is defined as a temporal trace function that can return the duration of an event even if it is discontinuous, because it returns the total minimal interval of duration by identifying the initial and final moments of the event. Any subinterval of the covering time of a maximally continuous event is the temporal trace of a subpart of such an event. The covering time of an event with a degree of continuity less than maximal has at least a gap in it, i.e. a subinterval which is the temporal trace of no subevent of $e$, cf. (4).

(4) $\quad \mathrm{F}_{\text {Continutity }}(\mathrm{P})(\mathrm{e})<1 \rightarrow \forall \mathrm{e}\left[\mathrm{P}(\mathrm{e}) \rightarrow \exists \mathrm{t}\left[\mathrm{t}<\tau^{C}(\mathrm{e}) \wedge \neg \exists \mathrm{e}^{\prime}\left[\mathrm{e}^{\prime}<\mathrm{e} \wedge \mathrm{t}=\tau\left(\mathrm{e}^{\prime}\right)\right]\right]\right]$

\footnotetext{
${ }^{1}$ Tatevosov rejects the label 'pluractionality' for the case of plurality he describes. However, the objection he raises concerning the singularity of the participants in the event, applies to event-external pluractionality, and on the contrary, supports a characterisation as event-internal plurality. Another objection he raises is that -kala- does not produce the full spectrum of readings typically associated with pluractionality. Note that having a partial spectrum is rather the standard case for a (pluractional) affix, and that the readings produced by -kala- mentioned in the paper are typical forms of diminutive event-internal plurality. It is not clear how well his approach generalises to cases where more than one dimension is affected at the same time, quite a common situation in event-internal plurality.
} 
Crucially, the definition of continuity relies on the possibility for $\tau^{C}(e)$ of identifying the initial and final moments of $e$. This is quite a standard assumption if one has a continuous event to start with, cf. [5], and gaps are subsequently added to its trace, whereas the reverse order of action is problematic, i.e. identifying the event by taking away gaps in an interval, as discussed below. Assuming a VP modifier status for -kala-, instead of V modifier, gets around the problem of carving out a subevent $e^{\prime}$ in an event $e$ with gaps as in (4). But no evidence nor explicit motivation for the VP level of modification are provided. The data show that $-k a l a-$ combines with the verb stem before perfective affixes and verb inflection, which is common for aspectual affixes.

Another way of characterising single events with an internal form of multiplicity has been explored by Tovena, in single and joint work, w.r.t. a class of Italian and French verbs derived by suffixation, e.g. It. mordicchiare (nibble), tagliuzzare (cut into small pieces). Plurality, she argues, arises from distributing over the cells of a cover applied to a participant. The levels of the event $e$ and of the plurality of phases $e^{\prime}$ are specified separately in (5), the plurality is given an explicit status at event level through groupification, and the equation $e=\uparrow e^{\prime}$ links levels in the representation. From this, we can work out that the suffix -icchi contributes a constraint like in (6) on the internal structure of the event.

$$
\| \text { mordicchiare } \|=\lambda x \lambda y \lambda e[(\operatorname{mordicchiare}(e) \& \operatorname{Ag}(e, y) \& \operatorname{Pat}(e, x)) \Leftrightarrow
$$
$\exists e^{\prime}\left({ }^{*}\right.$ morderePhase $\left.\left.\left(e^{\prime}\right) \& e=\uparrow e^{\prime} \&{ }^{*} \mathrm{Ag}\left(e^{\prime}, y\right) \&{ }^{M} \operatorname{Pat}\left(e^{\prime}, x\right)\right)\right]$

$$
\begin{array}{ll}
\text { a. } & \| \text { mordere } \|(\text { bite })=\lambda x \lambda y \lambda e[\operatorname{mordere}(e) \& \operatorname{Ag}(e, y) \& \operatorname{Pat}(e, x)] \\
\text { b. } & \|-i c c h i\|=\lambda O\left[\lambda x \lambda y \lambda e \left[( O ( x ) ( y ) ( e ) ) \Leftrightarrow \exists e ^ { \prime } \left({ }^{*} \operatorname{VPhase}\left(e^{\prime}\right) \& e=\uparrow e^{\prime}\right.\right.\right. \\
& \left.\left.\left.\&{ }^{*} \operatorname{Ag}\left(e^{\prime}, y\right) \&{ }^{M} \operatorname{Pat}\left(e^{\prime}, x\right)\right)\right]\right]
\end{array}
$$

Two operations are exploited to bring about the distribution effect within one event: i) at least one participant is fragmented via a mass role ${ }^{M} \mathrm{R}[6]$, and ii) this role is related to a predicate of phases that denotes in a plural domain, but it is the event predicate that is the basic one and that assigns roles to the participants. ${ }^{2}$ Tovena shows that the verb does not make phases accessible at discourse referent level so they cannot be counted, the cardinality of the plurality cannot be compared even when left unspecified, and there are thematic restrictions that do not apply to pluralities of other levels. The proposal in (5) generalizes by considering property scales measuring an abstract dimension instead of physical entities, e.g. the vol-

\footnotetext{
${ }^{2}$ The parts of the participant work as the distributive key, whose plurality becomes visible only within the event, via the mass role ${ }^{M} \mathrm{R}$. This role uses a cover necessarily weaker than the cover that has the atom as its unique cell.
} 
ume of a physical entity, as well as paths and scales associated to the event because of implicit arguments. In the canonical case, the unfolding of the event is measured by adjacent isomorphic transitions of the theme along a scale related to the event by Krifka's [5] Movement Relation. Whereas, in event-internal pluractional verbs, the correlation between a dynamic predicate and a form of gradability is disrupted. Fragmenting means to cancel the homomorphism between the mereological structures of (one or more) scales and the event.

Both proposals are about single events that exhibit a form of plurality and describe a situation as non canonical. They share the view that the source of multiplicity is to be sought somewhere in the unfolding of the event, and differ in the conception of plurality invoked.

\section{Grounding phases}

Grounding phases on time raises several problems. First and foremost, total absence of discontinuity - i.e. strict continuity - is generally not enforced in canonical events described by unmodified predicates and cannot be taken as a discriminating criterion. Let's call disc-V the class of verbs describing discontinuous events. The type of subevents that distinguish events of the $d i s c-\mathrm{V}$ type from canonical ones are no trivial gaps where the applicability of the predicate to the interval is suspended, but constitutive parts of the event type under definition. They are not a zooming-in effect. Let's call them lulls. Since they are a required component, the trace function applied to the event must return an interval that contains them too, otherwise the event is not of the disc-V type. This is the interval Tatevosov called covering time and that corresponds to the minimal convex interval that has the temporal trace of the $\mathrm{V}$ type event as subinterval.

Once we acknowledge that lulls are definitional for disc-V type of events, strictly speaking, there is no temporal discontinuity anymore, hence no source of multiplicity. In this respect, the characterisation of - kala- in Chuvas as VP modifier is instrumental, because it allows one to have what looks like a single discontinuous event that instantiates $e$ in (4) but is not directly defined as an event in itself. Yet, $\tau^{C}$ is assumed to correctly identify its initial and final moments, and it is the original property of event that is used to vouch for the composition of all the relevant discontinuous subevents into one event. But why invoking a property that needed to be modified precisely because it was not able to adequately characterise the event under examination? The general question is whether and how 
can we properly define/identify subevents other than by using temporal intervals, which by themselves do not define event properties.

Furthermore, what said for the event applies to subevents. Either, subevents are of the $d i s c-\mathrm{V}$ type and thus contain lulls, which means that disc- $\mathrm{V}$ events cannot be made of just two disc-V subevents, because these subevents themselves must contain lulls. ${ }^{3}$ We have a sorites paradox of the heap type here. Or, being two continuous subevents as Tatevosov assumes, they cannot be of disc-V type. They actually are of the $\mathrm{V}$ type. But this is a way to stipulate that all V-type subevents cannot cluster somewhere within the temporal trace of a disc-V event. Moreover, lulls cannot last too long lest the connectedness of the event is jeopardised. Something should be said about what prevents discontinuity from disrupting the event in (1a) into a collection of distinct events of eating (parts of) the same apple.

Let us look at (1a) with the insight that taking out lulls from the temporal trace of a disc-V event gives us the trace of a V-type event in its canonical realisation, and explore the alternative possibility of grounding discontinuity on a measure of increment. If events of the disc-V type must have discontinuous subevents, i.e. phases, this means that there must be some subevent(s) in which no part of the apple is in a $\theta$ relation with the event and the event's temporal progression is not suspended. As said above, events described by event-internal pluractional verbs are perceived as noncanonical instantiations of an event type. Instead of using the problematic notion of continuity, the notion of non-canonical event can be defined as the case of an event exhibiting localised losses of mapping-to-subobjects (MSO [5]) for a $\theta$ role whose corresponding $\theta^{\prime}$, which would be assigned by $\mathrm{V}$ to the same entity if the event where realised (or described as realised) in its canonical form, does have MSO. This localised effect is characterised by saying that a disc- $\mathrm{V}$ event must contain subevents that are $\mathrm{V}$ eventsin all of which $\theta$ satisfies MSO - and subevents that are not, and therefore are lulls. Generalising, i) the $\theta$ assigned by the pluractional- $V$ verb to the object is the closure of $\theta^{\prime}$ under sum formation of the object, where $\theta^{\prime}$ is the role assigned by simplex $\mathrm{V}$ to the object, and ii) $\theta^{\prime}$ has MSO and MO (mapping-to-object), while $\theta$ has not. The mapping must be extended to include measures used in the definition of event incrementality.

Discontinuity comes out from a modification of a property of a $\theta$ role instead of using a temporal definition. The multiplicity of phases perceived in the event is anchored in the parts of an entity/value relevant to the conceptualisation of the event and not in its temporal interval. The non-canonical event is characterised as a predictable modification of the

\footnotetext{
${ }^{3}$ Recall Lasersohn's cardinality constraint $n \geq 2$ endorsed by Tatevosov.
} 
semantic characterisation of the canonical form. This modified semantic characterisation matches the understanding that we are dealing with a class of verbs that have morphologically derived forms. The approach defended in this paper provides explicit motivation for the disequation $\mathrm{P} \neq \mathrm{V}$ for event-internal pluralities that Lasersohn had to stipulate. As we have seen, the nature of the event, i.e. its being non-canonical in a way that allows the expression of a local form of plurality, does not lend itself to a recursive definition, and phases cannot be described by the predicate that describes the event. This impossibility may be seen to lurk behind Tatevosov's requirement that subevents be continuous. Next, the issue of the duration of lulls, but not of their presence, boils down to the general, albeit non-trivial, issue of defining continuity for an event.

The complexity of the issue of where the pluractional marker does its modification highlights the fact that, as we just said, the event property used for describing the pluractional event cannot be the same as that used to describe phases, but also that the modification of the verb has consequences at various levels. On the one hand, properties of the theta roles assigned by the verb naturally belong to the content of the verb, and altering them is tantamount to modifying the ingredients of the aspectual characterisation of the verb, the so-called lexical or inner aspect. On the other hand, aspectual consequences spread in the whole VP up to sentential level. A change in the nature of the event description is the most salient output of the use of a pluractional form. We can add two language specific pieces of data from Italian and French to this discussion against an analysis of event-internal pluractional markers as VP modifiers. These languages use evaluative suffixes to form pluractional verbs of the eventinternal type. ${ }^{4}$ First, evaluative suffixes can form denominal pluractional verbs, beside deverbal ones, see (7). Hence the impact of the suffix must be assessed also below VP level.

a. Italian: sorseggiare (sip) $\leftarrow \operatorname{sorso}_{N}$ (sip)

b. French: pointiller $(\operatorname{dot}) \leftarrow$ point $_{N}($ dot $)$

Second, the suffix can affect the conjugation of the verb in deverbal cases, as discussed in [14]. Simplex verb forms may belong to any class of conjugation, whereas the derived pluractional forms all belong to the first class, e.g. 3rd conjugation tossire (cough) >1st conjugation tossicchiare. Evaluative suffixes bring about the same effect of normalisation on nouns.

\footnotetext{
${ }^{4}$ This word-formation option is available in other Romance languages, although with varying degrees of productivity. Evaluative morphology typically belongs to the nominal domain, but has a variety of uses [4].
} 
All modified nouns belong to the broadest inflection class, independently of the class of the base, e.g. poeta (poet) vs. poetino.

\section{On phases and minimal units}

In a plurality, phases are parts described all by the same predicate and endowed with some form of atomicity, that makes it possible for us to appreciate their multiplicity but does not warrant their identification. Thus, they cannot be counted, e.g. a claim of victory like Luisa ha mordicchiato più di Daniele (Luisa nibbled more than Daniele) cannot be foiled by replying No, perché lui è più veloce (no, because he is faster) with the intention of saying that he gave more little bites in the same time span. It is far from clear that a minimal cardinality can be defined unambiguously for these verbs, pace Tatevosov and Lasersohn. Again, the situation hints at a sorites paradox, where the beginning of a sorites series does not coincide with the beginning of a series. There may be a cut-off separating nibbling from biting when going in this direction - as one may concede that an event of two little bites is still a nibbling, yet an event with one is certainly not. There isn't necessarily a cut-off when going the other way. The issue is more complex than just having two series with non-coinciding beginnings. It involves the status of the units. A plurality of phases described by an event predicate $\mathrm{P}$ is homogeneous because phases are not identifiable, but $\mathrm{P}$ is not properly cumulative for reasons we have seen. First, not just any set of phases is an instance of a $\mathrm{P}$ event, in particular the singleton set is not. Second, given what we said on subevents in section 3 , only subevents understood as bigger than single phases can be properly called subevents of the same event type $\mathrm{P}$ and be added in a series. Third, lulls are subintervals on which we do not want to define subevents. In sum, it appears that cumulativity, and divisivity for that matter, are properties of events and are standardly tested by working with subevents that can qualify as events, not with phases.

Event-internal pluractionals are reminiscent of semelfactives, e.g. jump, cough, in their iterated/activity reading, but should be kept separate. Pluractional verbs may be derived from semelfactive bases, but they no longer have a semel use, cf It. tossire-tossicchiare (cough). In order to expose the differences, let's first recall some claims about semelfactives. Semelfactives are dynamic, as they can occur in the imperative form, and atelic instantaneous, as they combine with punctual adverbs, according to Smith [10], and their peculiarity is the absence of change. In the light of the discussion in section 2 , this means that the realisation of the event 
does not modify the preconditions, and iteration without gaps is possible as a consequence. As for the activity reading specifically, Rothstein [9] points out that semelfactives are event predicates that denote extended events, as they combine with duration adverbs, take progressive form and are said to induce the imperfective paradox. As for the single event reading, Rothstein, who takes semel verbs to be telic interval predicates related to homonymous activity predicates, is keen to recall that Smith herself acknowledges that semelfactive are events conceptualised as instantaneous though they take time to reach a completion in reality. Thus, semelfactives denote events that have internal structure - as opposed to achievements, which are analysed as near-instantaneous changes from $\neg \phi$ to $\phi$. For Rothstein, the occurrence in the progressive and the imperfective paradox is linguistic evidence for such a structure. Taking scores, we can observe that the combination of being instantaneous and allowing seamless sequences makes the activity reading of semelfactives look like a plurality of phases. The possibility of spatio-temporally locating events in the semel reading or units of events in their activity reading, makes these events and units different from phases and clearly sets apart semelfactives from pluractional verbs.

Events denoted in semel uses may be complex, but so do phases. The series of movements that must occur as part of an event denoted by a semelfactive, in Rothstein terms, are constitutive of its internal structure. It is a single instantiation of this structure that Rothstein takes as paralleling the minimal parts of an activity in Dowty's [2] terms, i.e. the smallest events in $\mathrm{P}$ that count as events of $\mathrm{P}$. The difference with ordinary activities, I suggest, is in the non-arbitrary way of dividing minimal events/units that is peculiar to semelfactives. We can see activities as characterised by cycles of parts, but in general their cycles do not have specific first/prominent elements, whereas in semelfactives they do. Rothstein assumes that activities denote in a domain where minimal events are not in an atomic set but in a singular set, whereas, in the semelfactive use of a predicate, a natural atomic function picks out the atomic set. The difference is that a singular set contains minimal singular but overlapping entities, and this, in Rothstein's words, means that two minimal events of walking may overlaps but two minimal events of jumping will not. The point is delicate and we do not need to take a stand on it. For our concerns, it suffices to assume that the minimal event in a singular set may have structure inside, but that no part in it qualifies as the beginning or the apex of the cycle, contrary to the minimal event in an atomic set. The recursion of the distinguished part signals the completion of a cycle, what precedes or follows it qualifies 
as part of the cycle. I won't try to define what counts as a full cycle. What matters is that the prominent part may act as the identifier for the whole event, as usual with achievements, and be relevant to count units/cycles that are viewed as events. Since the whole cycle is short, one-cycle events may be conceptualised as instantaneous.

In short, first, the identifying capacity of a minimal unit is an artefact in an activity and cannot be used to count events. It is the series that we count, leaving aside the important but independent issue of its continuity. Second, the combination of having well defined minimal units, which are perceived and characterised as such by a language, and having output conditions that meet input conditions, which amounts to what has been dubbed as absence of change in the literature, are the ingredients for the double behaviour of semelfactives. Minimal units are events. Semelfactive predicates denote single events in the semel use, and sums of events in the activity use. Minimal events can be added one at the time and yield an incremental process. Third, by contrast, event-internal pluractionals share the second ingredient at phase level, but crucially differ with respect to the first. Phases may still be viewed as cycles containing a prominent part that makes it possible to see a multitude, but are not individually mapped onto events in the hierarchy of Cusic, hence cannot be counted. They do not partake in the same event type as the grouped plurality they form.

\section{Conclusion}

Phases of a discontinuous internally plural event appear as a result of altering a $\theta$ relation of a canonical event description in a way that reduces cohesion. More specifically, at least one theta role lacks a property that is found in the description taken as canonical. The loss of MSO has to do with multiplication, hence it is coupled by a loss of MO. The same pattern of modification can be used to create new verbs from nominal bases.

Phases are viewed as the reflect of the application of the predicate to the parts of the participant demoted to a sum that works as the distributive key. This gives them a form of atomicity sufficient for event-internal plurality. But the modification makes the recursive application of the event property impossible, and phases do not qualify as minimal units of the event they are part of. Therefore, semelfactives and event-internal pluractional verbs are two different types of predicates. 


\section{Bibliography}

[1] David Cusic. Verbal plurality and aspect. PhD thesis, University of Stanford, 1981.

[2] David R. Dowty. Word meaning and Montague grammar. Reidel, Dordrecht, 1979.

[3] Wolfgang Dressler. Studien zur verbalen Pluralität. Osterreichische Akademic der Wissenschaft, Phil-Hist, Wien, 1968.

[4] Daniel Jurafsky. Universal tendencies in the semantics of the diminutive. Language, 72:533-578, 1996.

[5] Manfred Krifka. The origins of telicity. In Susan Rothstein, editor, Events and Grammar, pages 197-235. Kluwer, Dordrecht, 1998.

[6] Fred Landman. Events and plurality. Kluwer Academic Press, Dordrecht, 2000.

[7] Peter Lasersohn. Plurality, conjunction and events. Kluwer Academic Press, Dordrecht, 1995.

[8] Paul Newman. The classification of Chadic within Afroasiatic. Universitaire Pers., Leiden, 1980.

[9] Susan Rothstein. Structuring events. Blackwell Publishing, Oxford, 2004.

[10] Carlota Smith. The parameter of aspect. Studies in Linguistics and Philosophy. Kluwer Academic Publishers, Dordrecht, 1991.

[11] Sergei Tatevosov. Pluractionality vs. discontinuity. In Proceedings of the Sixteenth Amsterdam Colloquium, pages 217-222, 2007.

[12] Lucia M. Tovena. A class of pluractional verbs in Italian and French. handout, Semantics beyond set theory, Paris 25 October 2007, 2007.

[13] Lucia M. Tovena. Pluractional verbs that grammaticise number through the part-of relation. Proceedings of Going Romance 2008, to appear.

[14] Lucia M. Tovena. Issues in the formation of verbs by evaluative suffixation. Proceedings of XLIII Congresso della Società di Linguistica Italiana, to appear.

[15] Lucia M. Tovena and Alain Kihm. Nibbling is not many bitings in French and Italian: A morphosemantic analysis of internal plurality. In Proceedings of the 34th Annual Meeting of the Berkeley Linguistics Society, 2008. 